\title{
The effects of different attentional focus on poststroke gait
}

\author{
Sun-Ae Kim, Young Uk Ryu, Hwa Kyung Shin* \\ Department of Physical Therapy, College of Bio and Medical Science, Daegu Catholic University, Gyeongsan, Korea
}

Attention may influence the motor performance of poststroke patients. The attentional focus implies "where" attention is focused while performing a specific movement. Focusing attention on the inside of the body while performing a specific exercise is called an internal focus (IF) and focus on the external environment is called an external focus (EF). This study examined the effect of attention on the gait of patients with chronic stroke. Sixteen subjects voluntarily participated in the study. The subjects were guided to walk while maintaining the required attentional focus, which is control, IF, and EF condition. Dependent variables were as follows: step length, stride length, step width, 10-m walk time and weight distribution on the paretic limb. The results are as follows. First, the step length and the stride length were observed to be longer in the EF condition. Second, 10-m walk time was faster in EF condition. Third, under the EF condition, more weight was applied to the paretic limb. We suggest that EF instruction useful to improve the poststroke gait.

Keywords: Attention, Gait, Hemiplegia, Stroke

\section{INTRODUCTION}

Stroke can be caused by blockage of the blood supply or sudden bleeding in the brain (McIntyre and Mitchell, 2009). Stroke results in physical difficulties such as neurological problems and hemiplegia. Physically, muscle weakness, abnormal muscle tone, abnormal movements patterns, balance problems, and impaired weight shifting may occur (Latham et al., 2005). In particular, a person may temporarily or permanently lose normal postural control mechanisms after brain injury. Apparently, it would not be possible for a person to automatically carry out the tasks that are automatically processed, for example, maintaining a standing posture or gait (Lord et al., 2004; Patterson et al., 2007). Apparently, the consciousness and slow reaction, which are influenced by attention are achieved (Franceschini et al., 2009).

Most of the hemiplegic patients with stroke shift the center of gravity to the nonparetic legs and produce an asymmetric force in the lower limb. This asymmetric motor control of the lower limb may lead to asymmetric gait (Stojanović and Djurasić, 2013). Compared to the healthy adults, the gait in patients with stroke leads to the reduction in the stride length and the gait speed. In particular, the characteristics such as the difference in stride length, the difference in step length between paretic and nonparetic limb, and the shorter stance phase in the paretic limb appear. Stroke patients require long-term rehabilitation programs, of which treatments for gait disorder require significant efforts (Guzik et al., 2018).

Most of the recent studies have demonstrated that attention may influence motor performance (van Abswoude et al., 2018). The attentional focus implies "where" attention is focused while performing a specific movement. The criterion for attention is the body of the performer. Focusing attention on the inside of the body while performing a specific exercise is called as internal focus (IF) and focus on the external environment is called as external focus (EF).

In the past, studies have shown that EF is generally more effective on motor performance than IF (Chiviacowsky et al., 2010; Wulf et al., 2009). A great deal of research on balance control and motor learning demonstrates the benefits of EF (Parreira et al., 2013). Furthermore, a particular population, such as the elderly, Parkinson patients, and ankle sprain patients, revealed similar benefits (Chiviacowsky et al., 2010; Wulf et al., 2009). The advantage of the EF was also observed in gait condition. Studies in-
${ }^{*}$ Corresponding author: Hwa Kyung Shin (D https://orcid.org/0000-0003-3876-0710 Department of Physical Therapy, College of Bio and Medical Science, Daegu Catholic University, 13-13 Hayang-ro, Gyeongsan 38430, Korea E-mail: hkshin1@cu.ac.kr

Received: June 1, 2019 / Accepted: July 12, 2019
This is an Open Access article distributed under the terms of the Creative Commons Attribution Non-Commercial License (http://creativecommons.org/licenses/by-nc/4.0/) which permits unrestricted non-commercial use, distribution, and reproduction in any medium, provided the original work is properly cited. 
volving patients with multiple sclerosis, Parkinson disease, and the elderly have shown improvement in gait quality when using an EF (de Melker Worms et al., 2017; Shafizadeh et al., 2013).

It is reported that the attentional focus affects the balance or gait, and in general, the EF is mainly on the improvement of motor performance. However, few studies have employed attentional focus on the dynamic condition in patients with chronic stroke. The reason for the need to study the attentional focus is based on the hypothesis that it can be used as an indispensable technique for exercise rehabilitation in clinical practice. Therefore, this study examined the effect of different attentional focus on the poststroke gait. It is proposed that the use of a particular focus instruction will be beneficial for gait rehabilitation in stroke patients.

\section{MATERIALS AND METHODS}

\section{Participants}

The subjects who had been diagnosed with stroke through computed tomography or magnetic resonance imaging and who had passed 6 months or more after stroke onset were selected for the present study. The selection criteria included a score of 24 or more on the Korean version of Mini-Mental State Examination (K-MMSE), capable of understanding the directions of the researcher and walk independently without assistive devices. Among the selected subjects, 16 subjects (11 males and five females) voluntarily agreed to participate in the study and completed the experiment. This study was carried out with research approval by The Daegu Catholic University Review Board (CUIRB2019-0045).

\section{Experimental procedure}

First, the subjects were tested for the Berg balance scale to assess balance and the modified Ashworth scale for evaluation of motor function. The subjects were guided to walk while maintaining the required attentional focus. The three focus conditions were the control, IF, and EF. In the control, the subject was not provided with any instructions about the attentional focus. In the IF, the subject was instructed to walk while concentrating on his/ her lower limb movements. In the EF, the subject was instructed to walk by focusing on the markers and lines drawn on the floor based on the symmetrical gait cycle of healthy adult. Subjects were instructed to walk at a comfortable pace by looking ahead in all state focus conditions.

Subjects walked a total of $14 \mathrm{~m}$ and walked on a $120 \times 54-\mathrm{cm}$ mat-shaped Gait Analyzer (Tech Storm Inc., Seoul, Korea) at a distance of $7 \mathrm{~m}$ from starting point. Step length, stride length, step width, and weight distribution were collected through a built-in sensor. For 10-m walk test, it measured the walking time that taking for a distance of $10 \mathrm{~m}$ except for the beginning of $2 \mathrm{~m}$ and the end $2 \mathrm{~m}$. In order to prevent falls, a physical therapist always walked close to the paretic side of the subject.

Subjects repeatedly walked three times in each of the three different focus conditions, thereby performing a total of nine walks. After one walk, one minute of rest was given, and after each condition, 5 min of rest was taken by the subjects. All the subjects performed the control condition first. Subsequently, in order to offset the order effect on the attentional focus, eight subjects were in the order of IF and EF, and the other eight subjects were reversed. The order of focus condition was randomly selected.

\section{Measurement variables}

Dependent variables were as follows: step length, stride length, step width, 10-m walk time, and weight distribution on the paretic limb.

The step length is defined as the distance between the first point of contact of a limb with the ground and the first point of contact of the limb on the opposite side when a foot is progressed. In patients with stroke, the step lengths of the paretic leg and nonparetic legs were measured separately. The stride length is defined as the distance covered by the combined step length of each limb during one gait cycle. Therefore, longer step length and stride length are related to a higher quality of gait. The step width represents the horizontal distance between both feet. In the case of stroke patients, the step width is relatively larger compared to the healthy population because of the typical circumduction gait. Therefore, smaller step width is related to a higher quality of gait. The timed $10-\mathrm{m}$ walk test is the time taken to travel $10 \mathrm{~m}$, which means that shorter the time, the faster the walk. During the stance phase, the weight distribution ratio of the paretic limb represents the percentage of the weight on the paretic limb during gait. The larger the weight distribution ratio on the paretic limb, the more weight distribution occurs on the paretic limb.

\section{Statistical analysis}

The IBM SPSS Statistics ver. 19.0 (IBM Co., Armonk, NY, USA) was used for statistical analysis in this study. One-way repeated analysis of variance was used to analyze the significant differences between attentional focus conditions, which are independent variables for five dependent variables. LSD was used for post boc analysis. The statistical significance level $\alpha$ was set at 0.05 . 


\section{RESULTS}

\section{General characteristics of participants}

The general characteristics of the subjects are shown in Table 1.

\section{Step length}

Statistical significance was found for the step length of the paretic limb $(F=8.89, P<0.05)$. Post hoc indicates that $\mathrm{EF}$ is significantly larger than other conditions (Fig. 1A). Statistical significance was found for the nonparetic side $(F=6.64, P<0.05)$. Post hoc analysis shows that $\mathrm{EF}$ is significantly larger than other conditions (Fig. 1A).

\section{Stride length}

Statistical significance was found for stride length $(F=12.26$, $P<0.05)$. Post hoc analysis shows that $\mathrm{EF}$ is significantly larger than other conditions (Fig. 1B).

Table 1. General characteristics of participants

\begin{tabular}{lc}
\hline Variable & Mean \pm SD (range) \\
\hline Sex, male:female & $11: 5$ \\
Age (yr) & $49.81 \pm 8.16(39-66)$ \\
Height (cm) & $167.25 \pm 6.78(151-178)$ \\
Weight (kg) & $63.94 \pm 9.81(50-80)$ \\
Time since stroke (mo) & $27.63 \pm 11.64(10-45)$ \\
Paretic side, right:left & $9: 7$ \\
MAS (/48) & $37.06 \pm 6.80(25-46)$ \\
BBS (/56) & $42.75 \pm 7.07(34-55)$ \\
MMSE-K (/30) & $27.56 \pm 1.413(25-29)$
\end{tabular}

SD, standard deviation; MAS, motor assessment scale; BBS, Berg balance scale; MMSE-K, Korean version of Mini-Mental State Examination.

\section{Step width}

No statistical significance was found for step width $(P>0.05)$ (Fig. 1C).

\section{0-m walk time}

Statistical significance was found for 10 -m walk time $(F=13.12$, $P<0.05)$. Post hoc analysis shows that $\mathrm{EF}$ was significantly faster than other conditions (Fig. 2).

\section{Weight distribution}

Statistical significance was found for the weight distribution of the paretic limb $(F=9.02, P<0.05)$. Post hoc analysis shows that $\mathrm{EF}$ is significantly larger than other conditions (Fig. 3). Statistical significance was found for the weight distribution of the nonparetic limb $(F=8.89, P<0.05)$. Post hoc analysis shows that $\mathrm{EF}$ is significantly smaller than other conditions (Fig. 3).

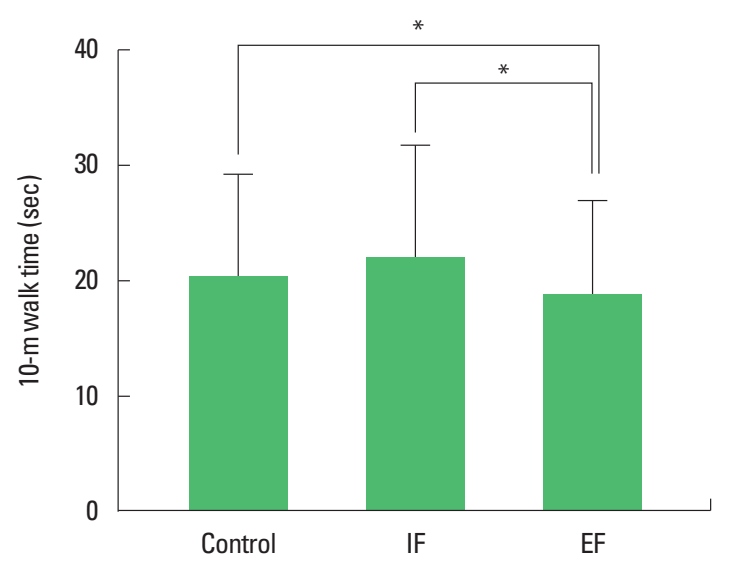

Fig. 2. Ten-meter walk time of poststroke gait as different of attentional focus. IF, internal focus; EF, external focus. ${ }^{*} P<0.05$.
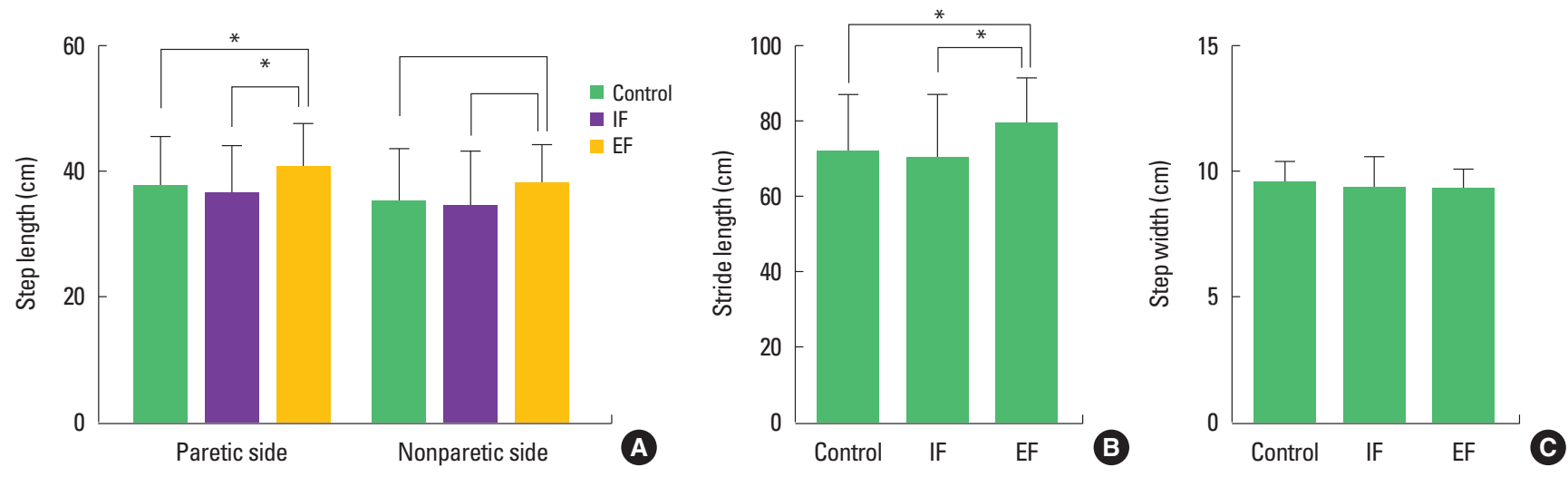

Fig. 1. Spatiotemporal parameter of poststroke gait as different of attentional focus. (A) Step length. (B) Stride length. (C) Step width. IF, internal focus; EF, external focus. ${ }^{*} P<0.05$. 


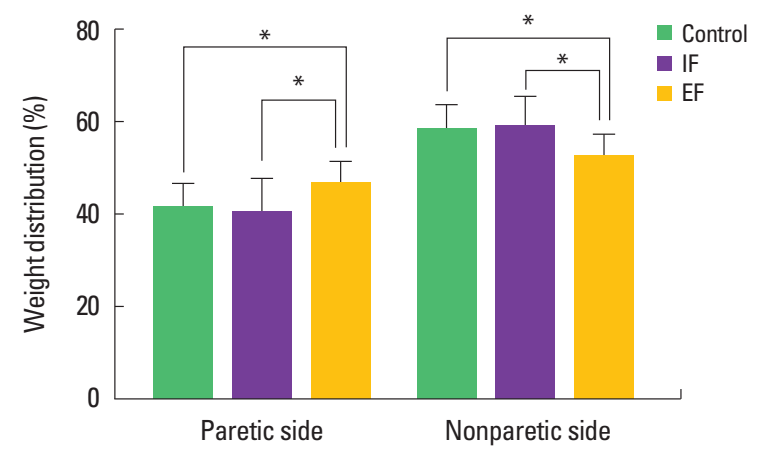

Fig. 3. Weight distribution of poststroke gait as different attentional focus. IF, internal focus; EF, external focus. ${ }^{*} P<0.05$.

\section{DISCUSSION}

The purpose of this study was to investigate the effect of attention on the poststroke gait. The subjects were asked to walk using three attentional focus conditions (control, IF, EF). The main results are as follows. First, the step length and the stride length were observed to be longer in the EF condition. Second, 10-m walk time was faster in $\mathrm{EF}$ condition. Third, under the $\mathrm{EF}$ condition, more weight was applied to the paretic limb. These results indicate that EF can improve gait in stroke patients. In this section, the results and clinical implications are discussed.

First, the step length and the stride length in EF condition were larger compared to the other two conditions, which signified that stroke patients are more likely to increase the spatial variables of gait by paying attention to the external environment. More interestingly, it also resulted in an increase in the step length of the paretic limb as well as the nonparetic limb. These results indicate that EF can improve the gait function of the paretic limb. Also, the subject walked significantly faster than other focus conditions in a timed 10-m walk test. Accordingly, instruction using EF can be considered to result in positive activation of the paretic limb in step length, stride length, and walk time.

The positive effects of EF on gait have also been demonstrated in other studies. The study by Shafizadeh et al. (2013) on patients with multiple sclerosis revealed that step length, stride length, gait speed, and gait energy consumption was significantly when using the EF. Beck et al. (2018) studied the effect of attentional focus on the gait of patients with Parkinson disease. The study demonstrated an increase in stride length by $90 \%$ when walking with EF training. The study of de Melker Worms et al. (2010) on older people revealed improvement in the spatial and temporal variables of gait when the EF was used.

Another important finding in the current study is that the EF during walking was more weight distributed on the paretic limb. Stroke patients usually transfer more weight to the nonparetic limb during walking and standing. In the present study, there was no significant difference between control and IF conditions, which implies that IF did not affect the asymmetry of weight distribution. However, patients with stroke transferred more weight on the paretic limb in the EF condition. These results show that EF can eventually shift the weight to the more paretic limb. Consequently, it is hypothesized that gait stability can be improved.

Few studies have shown that EF helps in weight distribution to the paretic limb during dynamic walking. However, there are many reports stating that $\mathrm{EF}$ is useful in static postural control. EF seems to reduce the postural sway during quiet standing compared to IF (Stins et al., 2015). The decrease in postural sway means that the posture is more stable, indicating that EF improved the static balance ability. Also, improvement of static balance implies a balance of both the feet on weight distribution, thereby supporting our results. Furthermore, a study by Wulf et al. (2009) on patients with Parkinson disease demonstrated a reduction in postural instability when EF was applied during quiet standing and walking. These results suggest that $\mathrm{EF}$ can improve the gait function attributable to the symmetry of weight distribution.

Further, EF was found to be effective in improving the step length, stride length, and increasing the weight shifting to the paretic limb. The possible mechanism for improvement in the gait quality in stroke patients by EF can be explained based on the constrained action hypothesis, which states that the IF interferes with effective motor control by making conscious control during the movement. On the contrary, EF was found to promote the automatic information processing of motor, thereby improving the motor control. In the present study, IF is thought to have intensely focused on the patient's own gait, and consequently, both the paretic and nonparetic limbs remained inadequately controlled. However, EF more likely rendered the paretic limb to automatically move, which in turn advanced automatic movement of the two legs, thereby resembling a normal gait pattern. As a result, weight distribution on the paretic side was increased, and finally walking speed was increased.

Johnson et al. (2013) showed that clinical physical therapists tend to use IF more often during gait training and that this approach hinders the automaticity of movement, motor learning, and retention. The present study provides a clinical implication of how to use attentional focus when physical therapists rehabilitate stroke patients. In other words, circumvention of the EF instruc- 
tion appears as useful to improve balance and walking in patients with stroke and promote the autonomy of the movement through the $\mathrm{EF}$ instruction.

The limitations of this study are as follows: First, it was difficult to generalize the results of the study in all stroke patients as we did not classify the subject's group by the brain lesion. Second, it was not possible to represent the effect of the attentional focus through continuous training by measuring only immediate effects. Therefore, further studies on the role of the attentional focus provided to learners should be continued to generalize the results of this study. It is proposed that scientific evidence for more efficient and concrete methods to improve walking in stroke patients should be continuously reported in connection with various variables.

\section{CONFLICT OF INTEREST}

No potential conflict of interest relevant to this article was reported.

\section{REFERENCES}

Beck EN, Intzandt BN, Almeida QJ. Can dual task walking improve in parkinson's disease after external focus of attention exercise? A single blind randomized controlled trial. Neurorehabil Neural Repair 2018; 32:18-33.

Chiviacowsky S, Wulf G, Wally R. An external focus of attention enhances balance learning in older adults. Gait Posture 2010;32:572-575.

de Melker Worms JLA, Stins JF, van Wegen EEH, Verschueren SMP, Beek PJ, Loram ID. Effects of attentional focus on walking stability in elderly. Gait Posture 2017;55:94-99.

Franceschini M, Carda S, Agosti M, Antenucci R, Malgrati D, Cisari C; Gruppo Italiano Studio Allevio Carico Ictus. Walking after stroke: what does treadmill training with body weight support add to overground gait training in patients early after stroke?: a single-blind, randomized, controlled trial. Stroke 2009;40:3079-3085.

Guzik A, Drużbicki M, Przysada G, Brzozowska-Magoń A, Wolan-Nieroda A, Kwolek A. An assessment of the relationship between the items of the observational Wisconsin Gait Scale and the 3-dimensional spatiotemporal and kinematic parameters in post-stroke gait. Gait Posture 2018;62:75-79.

Johnson L, Burridge JH, Demain SH. Internal and external focus of attention during gait re-education: an observational study of physical therapist practice in stroke rehabilitation. Phys Ther 2013;93:957-966.

Latham NK, Jette DU, Slavin M, Richards LG, Procino A, Smout RJ, Horn SD. Physical therapy during stroke rehabilitation for people with different walking abilities. Arch Phys Med Rehabil 2005;86(12 Suppl 2):S41-S50.

Lord SE, McPherson K, McNaughton HK, Rochester L, Weatherall M. Community ambulation after stroke: how important and obtainable is it and what measures appear predictive? Arch Phys Med Rehabil 2004;85:234-239.

McIntyre M, Mitchell EA. Longer term rehabilitation for patients following stroke: a literature review. Br J Neurosci Nurs 2009;5:34-38.

Parreira RB, Amorim CF, Gil AW, Teixeira DC, Bilodeau M, da Silva RA. Effect of trunk extensor fatigue on the postural balance of elderly and young adults during unipodal task. Eur J Appl Physiol 2013;113:19891996.

Patterson SL, Forrester LW, Rodgers MM, Ryan AS, Ivey FM, Sorkin JD, Macko RF. Determinants of walking function after stroke: differences by deficit severity. Arch Phys Med Rehabil 2007;88:115-119.

Shafizadeh M, Platt GK, Mohammadi B. Effects of different focus of attention rehabilitative training on gait performance in Multiple Sclerosis patients. J Bodyw Mov Ther 2013;17:28-34.

Stins JF, Emck C, de Vries EM, Doop S, Beek PJ. Attentional and sensory contributions to postural sway in children with autism spectrum disorder. Gait Posture 2015;42:199-203.

Stojanović B, Djurasić L. Predictive importance of index of asymmetry in recovery following stroke. Acta Chir Iugosl 2013;60:101-104.

van Abswoude F, Nuijen NB, van der Kamp J, Steenbergen B. Individual differences influencing immediate effects of internal and external focus instructions on children's motor performance. Res Q Exerc Sport 2018;89:190-199.

Wulf G, Landers M, Lewthwaite R, Töllner T. External focus instructions reduce postural instability in individuals with Parkinson disease. Phys Ther 2009;89:162-168. 